



\title{
Soft X-ray induced oxidation on acrylic acid grafted luminescent silicon quantum dots in ultrahigh vacuum
}



\author{
Yimin Chao, ${ }^{*, 1}$ Qi Wang ${ }^{1}$, Annette Pietzsch ${ }^{2}$, Franz Hennies ${ }^{2}$, and Hongjun $\mathrm{Ni}^{3}$ \\ ${ }^{1}$ School of Chemistry, University of East Anglia, Norwich NR4 7TJ, United Kingdom \\ ${ }^{2}$ MAX-Lab, Lund University, 22100 Lund, Sweden \\ ${ }^{3}$ School of Mechanical Engineering, Nantong University, Nantong 226019, P.R. China
}

Received 6 April 2011, accepted 20 May 2011

Published online 27 June 2011

Keywords core levels, quantum dots, silicon, XPS

*Corresponding author: e-mail y.chao@uea.ac.uk, Phone: +00 441603 593146, Fax: +00 441603592003

Water soluble acrylic acid grafted luminescent silicon quantum dots (Si-QDs) were prepared by a simplified method. The resulting Si-QDs dissolved in water and showed stable strong luminescence with peaks at 436 and $604 \mathrm{~nm}$. X-ray photoelectron spectroscopy (XPS) was employed to examine the surface electronic states after the synthesis. The co-existence of the $\mathrm{Si} 2 \mathrm{p}$ and $\mathrm{C} 1$ s core levels infers that the acrylic acid has been successfully grafted on the surface of silicon quantum dots. To fit the Si2p spectrum, four components were needed at 99.45 , $100.28,102.21$ and $103.24 \mathrm{eV}$. The first component at $99.45 \mathrm{eV}$ (I) was assigned to $\mathrm{Si}-\mathrm{Si}$ within the silicon core of the Si-QDs. The second component at $100.28 \mathrm{eV}$ (II) was from
Si-C. The third at $102.21 \mathrm{eV}$ (III) was a sub-oxide state and the fourth at $103.24 \mathrm{eV}$ (IV) was from $\mathrm{SiO}_{2}$ at $\mathrm{Si}$-QDs surface. With an increase in exposure to soft $\mathrm{X}$-ray photons, the intensity ratio of the two peaks within the $\mathrm{Si} 2 \mathrm{p}$ region $\mathrm{A}$ and $\mathrm{B}$ increased from 0.5 to 1.4 while the peak A intensity decreased, and eventually a steady state was reached. This observation is explained in terms of photon-induced oxidation taking place within the surface dangling bonds. As the PL profile for Si-QDs is influenced by the degree of oxidation within the nanocrystal structure, the inducement of oxidation by soft X-rays will play a role in the range of potential applications where such materials could be used - especially within biomedical labelling.
1 Introduction As the basic building block of semiconductor electronics, silicon is a widely available, comparatively cheap, ecologically friendly and technologically well developed material. Since 1990, when Canham [1] found silicon nanostructures were able to emit visible light, interest in nanoscale silicon structures has risen sharply throughout the wider scientific community [2-11]. Owing to their ability to emit red light and lack of toxicity $[12,13]$, silicon quantum dots (Si-QDs), are deemed to be one of the best candidates as bio-labels for applications in bio-imaging. However, their full potential faces a major barrier in respect of their water solubility. Unfortunately the popular alkylcapped Si-QDs are hydrophobic, and cannot be dissolved in water. Thus they are of limited use in a wide range of biological applications. Several groups have attempted to make the QDs water soluble by various routes [14-18]. Warner et al. [19] produced allylamine-capped Si-QDs using a Pt catalyst. The resulting Si-QDs were water-soluble and exhibited strong blue photoluminescence (PL) with a rapid rate of recombination. In contrast, $\mathrm{Li}$ and Ruckenstein [15] bound poly-acrylic acid (PAA) onto the Si-QDs surface by a UV induced graft method. These photo stable propionic acid (PA) terminated Si-QDs were alleged to have great potential in biological imaging. In a subsequent contribution by Sato and Swihart [16], water-dispersible PA-terminated Si-QDs were prepared by photoinitiated hydrosilylation. This work demonstrated that the Si-QDs size and corresponding PL emission colour could be controlled by varying the etching time and allowed a high density of carboxylic acid moieties to be used to covalently immobilize molecules containing amines groups, such as proteins $[20,21]$. Thus, the preparation of PA-terminated Si-QDs is one of more the promising approaches to generate bio-functional surfaces. A simplified synthesis method has been used to prepare samples of water-dispersible luminescent Si-QDs in this work. The acrylic acid grafted method has been adopted $[15,16]$, but 
a)


Figure 1 (online colour at: www.pss-a.com) (a) Schematic structure of acrylic-acid grafted Si-QD used in this work. The luminescent silicon core is crystalline with similar lattice parameters to bulk silicon. The carboxyl $(-\mathrm{COOH})$ group makes the Si-QDs water dispersible. (b) A photo of clear stable water solution of acrylicacid grafted $\mathrm{Si}$-QDs under UV light $(324 \mathrm{~nm})$. The solution remained transparent and without turbidity over several weeks.

the initial silicon quantum dot core was obtained by electrochemically etching a silicon chip with HF [22]. Figure 1a shows the schematic structure of the Si-QD product. With the carboxyl group $(-\mathrm{COOH})$ on the surface, the acrylic acid grafted Si-QDs possess better water solubility than previous alkylated Si-QDs [13]. Whereas, alkylated Si-QDs are hydrophobic, acrylic acid grafted $\mathrm{Si}-$ QDs are hydrophilic. A clear stable water solution of $0.4 \mu \mathrm{M}$ Si-QDs is shown in Fig. 1b. The solution remained transparent and without turbidity over several weeks. In this paper we report the results of the first study of the electronic structures of such water dispersible Si-QDs under exposure to soft X-ray radiation. The motivation behind this work is twofold: over the course of exposure the QDs are observed to undergo a photon-induced oxidation, based on the evolution of the Si2p core level, and so is of interest from a purely investigative perspective in furthering our understanding of their luminescence behaviour. Similar photon-oxidation effects have been observed in light-emitting porous silicon [23], germanium nanocrystals [24], alkylated silicon nanocrystals [25, 26], and crystalline silicon surfaces [27, 28], where the photon source was either a laser, UV, EUV/VUV, or X-rays. This work is of particular value owing to the ability to disperse acrylic acid grafted Si-QDs in water which may offer new routes towards bio-imaging applications within living systems. Oxidative effects are understood to play a critical role in the luminescence profiles of nanoscale silicon systems and so information relating the progression of oxidation through soft X-ray irradiation upon the observed emission bands will be useful where such structures may be used in X-ray radiation environments.

\section{Experimental}

2.1 Si-QDs preparation The detailed synthesis method has been described in previous published paper [29]; here we provide a brief description of the sample preparation. Photoluminescent silicon layers were formed by galvanostatic anodization of boron-doped $\mathrm{p}-\mathrm{Si}$ (100) oriented wafer $(10 \Omega \mathrm{cm}$ resistivity, Compart Technology, Peterborough, UK) in a 1:1 v/v solution of $48 \%$ aqueous HF and ethanol solution. The boron-doped p-Si (100) was chosen because of its high etching rate, hence the product rate $[30,31]$. The circular electrochemical etching cell $(1 \mathrm{~cm}$ diameter) was machined from polytetrafluoroethylene (PTFE). The silicon wafer was sealed to the base using a Viton $^{\mathrm{TM}}$ O-ring. The counter electrode was a piece of platinum wire coiled into loop to improve the uniformity of the current distribution, and the etching was carried out using KEITHLEY 2601 in constant current source mode. A layer of luminescent porous silicon was made at high current density $\left(5 \mathrm{~min}\right.$ at $\left.550 \mathrm{~mA} / \mathrm{cm}^{2}\right)$. The solution was decanted and the fluorescing porous silicon layer on the chip was transferred to a Schlenk flask and dried under the vacuum of a rotary pump for $2 \mathrm{~h}$. The air within a 1:99 v/v solution of acrylic acid and ethanol aqueous was purged by $\mathrm{N}_{2}$ bubbling for $2 \mathrm{~h}$. The solution was then added to the Schlenk flask containing $\mathrm{Si}$ chips (working under $\mathrm{N}_{2}$ protection). The Schlenk flask was tightly closed and subjected to ultrasonic dispersion for $50 \mathrm{~min}$ at $40^{\circ} \mathrm{C}$. The mixture was then poured into a polyethylene bottle and treated with $\mathrm{N}_{2}$ bubbling for $10 \mathrm{~min}$ to purge the air. Afterwards the mixture was inserted into an UV reactor and kept stirring at $50^{\circ} \mathrm{C}$ for $5 \mathrm{~h}$. This procedure is to change the $\mathrm{Si}-\mathrm{QD}$ s surface from hydrogen terminated (H-) to poly-acrylic acid terminated (PA-). After filtration the luminescent solution, ca $20 \mathrm{ml}$, was collected. After solvent evaporated in low vacuum line, a dry sample of $10 \mathrm{mg}$ was obtained.

2.2 Photoluminescence spectroscopy Photoluminescence (PL) spectra were acquired by PerkinElmer LS55 Fluorescence Spectrometer with the excitation from Xenon lamp at $310 \mathrm{~nm}$.

2.3 X-ray photoelectron spectroscopy X-ray photoemission spectroscopy (XPS) experiments were carried out with a Scienta R4000 analyser at beamline I511 of Max-lab in Lund [32], Sweden. For this purpose a few drops of suspension were cast onto gold film substrate. The film was immediately introduced into a load-lock attached to the ultra high vacuum (UHV) chamber in which the typical pressures were kept below $5 \times 10^{-10} \mathrm{mbar}$. All Si2p spectra were acquired with photon energy $150 \mathrm{eV}$, while $\mathrm{C} 1 \mathrm{~s}$ core level spectra were obtained with photon energy $347 \mathrm{eV}$. The binding energies were referred to the Fermi edge measured on a gold foil in direct electrical contact with the sample, and the energy resolution was $0.01 \mathrm{eV}$ at photon energy $150 \mathrm{eV}$.

3 Results and discussion The acrylic acid grafted luminescent $\mathrm{Si}$-QDs can be dried by removing residual solvent and re-dissolved in deionized (DI) water. The solution of Si-QDs in DI water showed a clear and strong red fluorescence under illumination by a hand held mercury lamp $(\lambda=254 \mathrm{~nm})$. The PL spectrum shows two peaks at $604 \mathrm{~nm}$ (red) and $436 \mathrm{~nm}$ (blue), respectively, see Fig. 2a. 


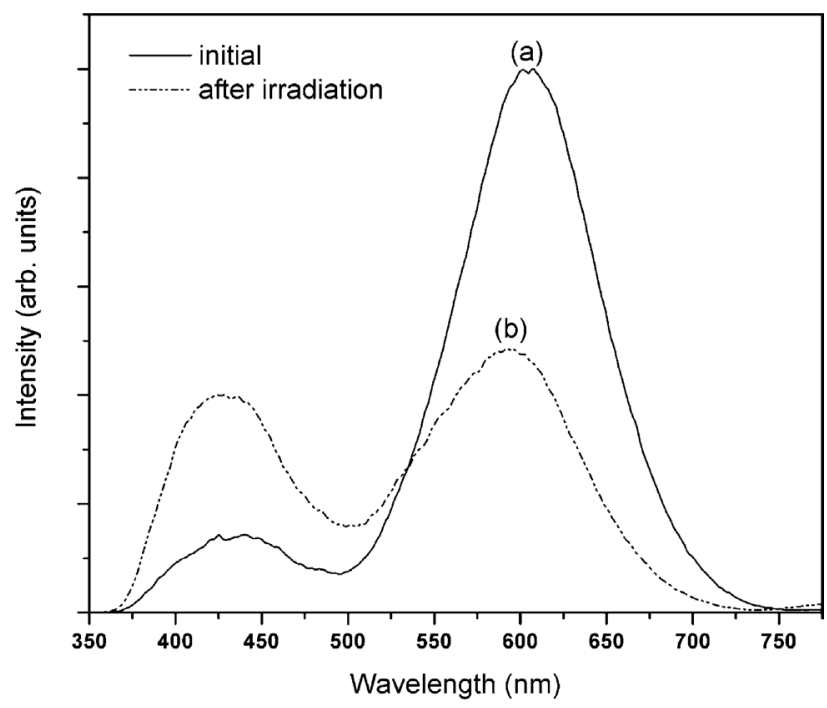

Figure 2 Photoluminescence spectra from acrylic acid grafted water dispersible silicon quantum dots. There are two peaks at 604 and $436 \mathrm{~nm}$, originated from un-oxidized and oxidized silicon, respectively. The PL measurement was performed by illuminating light at $\lambda=310 \mathrm{~nm}$. (a) Before the sample exposed to soft X-ray, red peak was the dominant; (b) after soft X-ray irradiation exposure, the blue peak was comparable to the red peak.

There are at least two plausible reasons for the observation of two emission bands. First, there may be a bimodal distribution of Si-QD sizes, giving rise to the two observed photon energies. Second, the two PL emission bands may result from different chemical states of $\mathrm{Si}$ : unoxidized $\mathrm{Si}$ such as $\mathrm{Si}-\mathrm{Si}$ bonds in the silicon core and oxidized $\mathrm{Si}$ atoms such as $\mathrm{Si}=\mathrm{O}$ bonds at the interface of core and grafted monolayer. We can discard the first possibility because no evidence of such a bimodal size distribution is present in our previous scanning tunnelling microscope (STM) or transmission electron microscopy (TEM) studies on alkylated Si-QDs [25, 33]. The mean size resulting from electrochemical etching would be expected to depend on the applied current density; therefore a narrow size distribution is quite expected. The second possibility is more likely since previous FTIR and photoemission spectroscopy measurements show that there is a small amount of oxide present in the samples $[22,25]$. The work of others also supports this interpretation $[34,35]$. When Si-QD is oxidized, the $\mathrm{Si}-\mathrm{Si}$ or $\mathrm{Si}-\mathrm{O}-\mathrm{Si}$ bonds are likely to weaken or break in many places because of the stress at the $\mathrm{Si} / \mathrm{SiO}_{2}$ interface [36]. A Si=O double bond is likely to be formed and stabilize the surface, since it does not require a large deformation energy. Such bonds have been suggested at the $\mathrm{Si} / \mathrm{SiO}_{2}$ interface [36]. In the literature [37], the PL emission from pure bulk silica is at $2.8 \mathrm{eV}(442 \mathrm{~nm})$, which is close to the blue peak from our samples at $436 \mathrm{~nm}$. Sham et al. [38] measured X-ray excited optical luminescence (XEOL) and X-ray emission spectroscopy (XES) on silicon nanowires and showed that the blue emission is associated with the silicon oxide layer on the samples. This suggests that the blue PL emission is from oxidized Si species and the red PL emission originates from unoxidized Si core, which is in agreement with the previous results from alkyl Si-QDs [26]. It should be noted that the PL colour will be dominated by the main peak $604 \mathrm{~nm}$ here, which is red. In other cases, if the peak at $436 \mathrm{~nm}$ is much stronger than red peak, the PL colour would be dominated by blue [25, 39-41]. Figure $2 b$ shows the PL after soft X-ray irradiation exposure $X=$ flux $\times$ time $=703 \mathrm{nA}$ min, which was obtained after the XPS measurement. Owing to the nature of this XPS apparatus setup, the X-ray spot remained on the same Si-QDs, thus we were not able to measure PL during the XPS experiments. Therefore, we can only compare PL spectra before and after XPS experiments. It is clear that the oxide related peak at $436 \mathrm{~nm}$ grew up to similar level as red peak. Because the blue peak is oxide related and the red peak is related to unoxidized $\mathrm{Si}$, this result, combined with the following XPS results, is strong evidence of oxidation induced by soft X-ray.

The surface electronic states were examined by employing high resolution XPS. Figure 3 shows high resolution XPS spectra over the Si2p and C1s core level energy regions from Si-QDs after the graft polymerization in $1 \% \mathrm{v} / \mathrm{v}$ acrylic acid monomer solution. To enhance the surface sensitivity, the spectra were collected at $20^{\circ}$ to normal emission with photon energies of 150 and $347 \mathrm{eV}$, respectively, and normalized to photon flux. In Fig. 3a, the Si2p spectrum was fitted with four mixed doublets and one Shirley background. The four components were at 99.45, 100.28, 102.21 and $103.24 \mathrm{eV}$, respectively. The first component at $99.45 \mathrm{eV}$ (I) is assigned to $\mathrm{Si}-\mathrm{Si}$ within silicon crystalline core of the $\mathrm{Si}-\mathrm{QDs}$. The second component at $100.28 \mathrm{eV}$ (II) is from $\mathrm{Si}-\mathrm{C}$. The third at $102.21 \mathrm{eV}$ (III) is a sub-oxide state and the fourth at $103.24 \mathrm{eV}$ (IV) from $\mathrm{SiO}_{2}$ at surface of Si-QDs, respectively $[42,43]$. The existence of a Si-C component here infers that the silicon atoms at the nanoparticle surfaces have changed from $\mathrm{H}$ - to a PA-termination and provides compelling evidence of the successful synthesis of acrylic acid grafted luminescent water dispersible Si-QDs [16]. A broadened peak at $103.24 \mathrm{eV}$ (IV) was observed, confirming the sample surface has been oxidized under the soft X-ray irradiation. The full width at half maximum (FWHM) of each specie can be obtained during the fitting. FWHM of specie IV was $1.7 \mathrm{eV}$, which is considerably broader than that of specie I $(\sim 1.1 \mathrm{eV})$. The component II at $100.28 \mathrm{eV}$ confirmed the existence of $\mathrm{Si}-\mathrm{C}$ on the surface. It should be observed that a $\mathrm{Si}-\mathrm{C}$ component was needed to fit the $\mathrm{C} 1 \mathrm{~s}$ spectrum as well. The $\mathrm{C} 1 \mathrm{~s}$ spectrum was fitted with four mixed singlets and one Shirley background. The three components were at 283.8, 284.92, 286.66 and $289.38 \mathrm{eV}$, see Fig. 3b. The first component (I) $\mathrm{C} 1 \mathrm{~s}$ peak, at binding energy $283.8 \mathrm{eV}$, can be ascribed to emission from core-level electrons of carbon atoms covalently bonded to the relatively electro-positive silicon (C-Si) [43-45]. The second peak (II), at binding energy $284.92 \mathrm{eV}$, can be ascribed to carbon bonded to either hydrogen or another carbon atom. The third component (III) of the $\mathrm{C} 1 \mathrm{~s}$ peak, at a binding energy $286.6 \mathrm{eV}$, is ascribed to adventitious carbon bonded to silicon, or oxygen from 

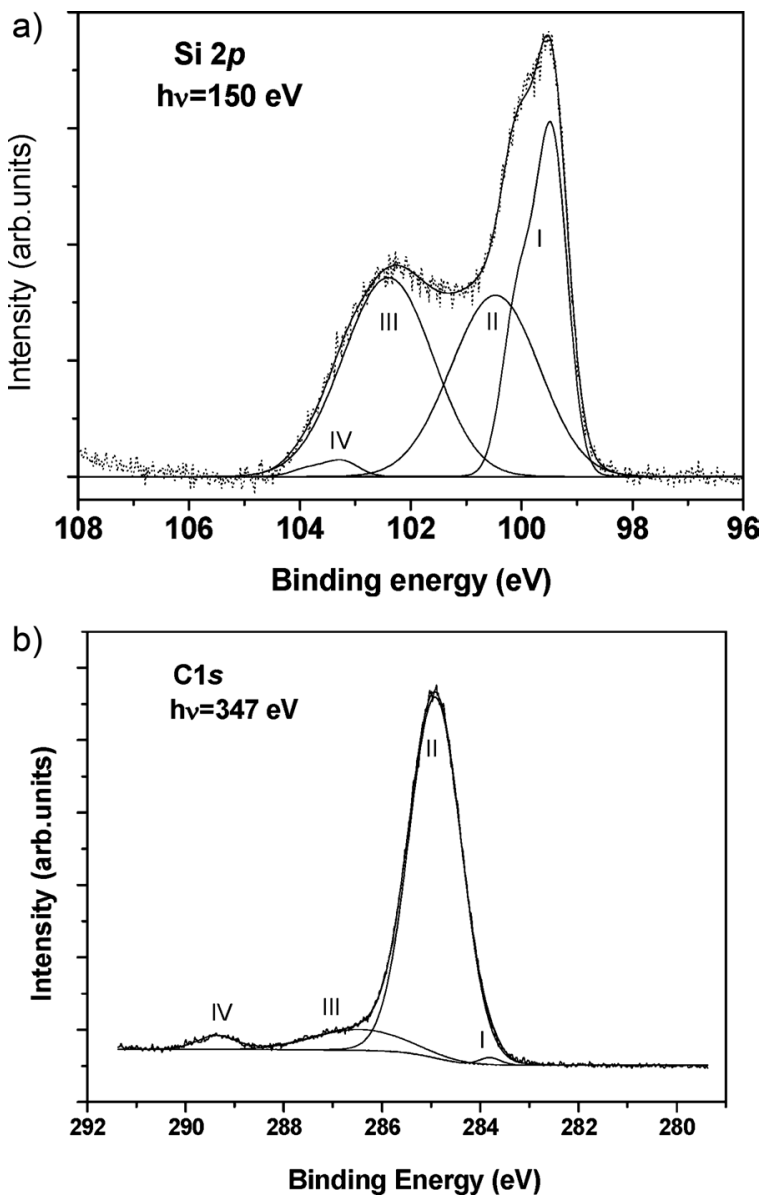

Figure 3 Core level XPS spectra obtained at $20^{\circ}$ to normal emission: (a) $\mathrm{Si} 2 \mathrm{p}$, the dotted line was experimental data which was fitted by four mixed doublets and one Shirley background: the four components at $99.45,100.28,102.21$ and $103.24 \mathrm{eV}$, respectively. These components were corresponding to the species: silicon within the core silicon crystalline (I), silicon-carbon bond (II), sub-oxide state (III) and silicon oxide (IV) on the surface of Si-QDs. (b) C1s was fitted by four mixed doublets and one Shirley background. The four components are: (I) $283.8 \mathrm{eV}$ from Si-C, (II) $284.92 \mathrm{eV}$ from C-C or C-H, (III) $286.66 \mathrm{eV}$ from adventitious carbon and (IV) $289.38 \mathrm{eV}$ from carbon in carboxylic acid group.

carbonaceous materials present in the laboratory environment, and/or from the transportation of samples to the XPS chamber [45]. The fourth (IV) distinct high binding energy at about $289.38 \mathrm{eV}$ is characteristic of the carboxylic acid group of the grafted acrylic acid polymer. The presence of this peak, together with published FTIR data confirms the existence of a surface grafted poly-acrylic acids [15, 29]. To further investigate the photon-induced oxidation, the evolution of the Si2p core level was monitored over the period of the X-ray irradiation. Figure 4 a shows the evolution of the $\mathrm{Si} 2 \mathrm{p}$ core level during a course of irradiation by $150 \mathrm{eV}$ soft $\mathrm{X}$-ray photons. Exposure is defined as $X=$ flux $\times$ time, from 0 to $703 \mathrm{nAmin}$. As one can see from the fittings in Fig. 3a, peak A is related to core silicon crystals and suboxide states while peak B is related to oxide and $\mathrm{Si}-\mathrm{C}$. The
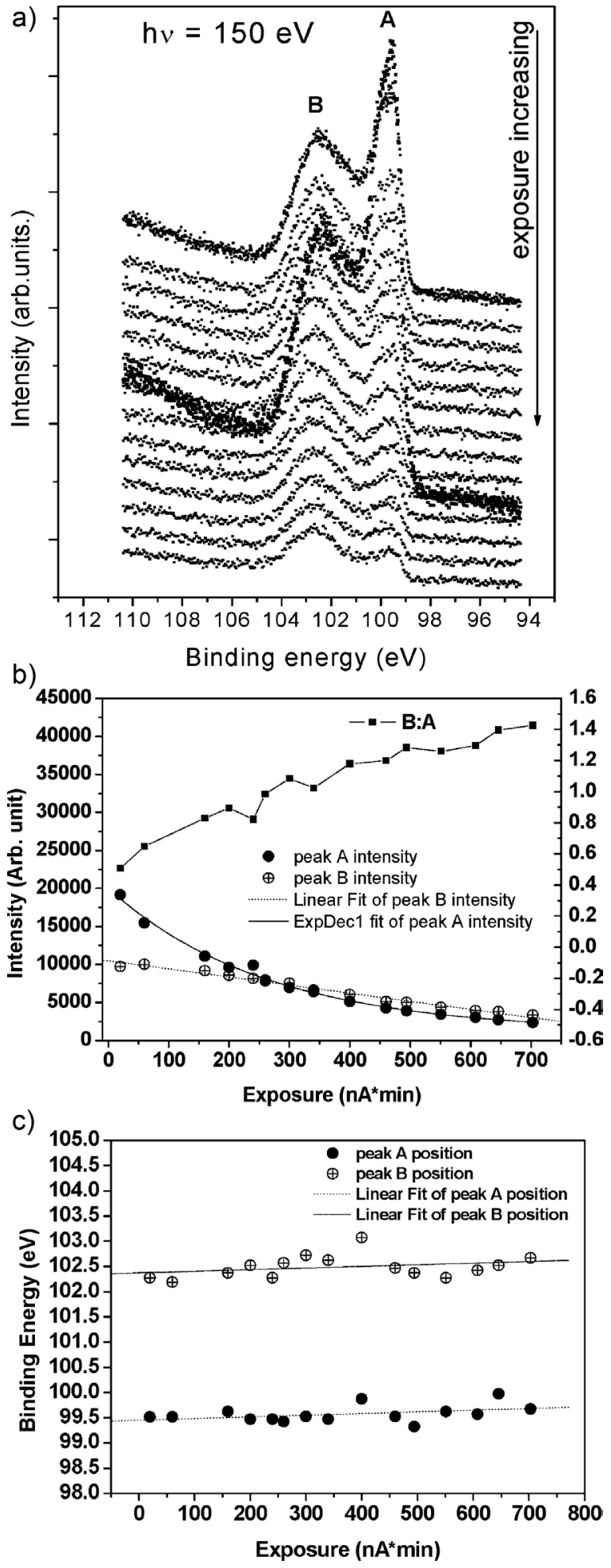

Figure 4 (a) The evolution of core level Si2p during the course of soft X-ray irradiation at photon energy $150 \mathrm{eV}$; (b) the intensity changing of two components before final steady state reached: the intensity of Si related peak decreasing faster than the intensity of oxide related peak during the course of exposure, and intensity ratio of B:A risen from 0.5 to 1.4 ; (c) no significant changes of peak positions observed during the irradiation course. 
intensities of the both Si2p peaks, A and B were observed to decrease with increasing exposure to radiation. From the raw data in Fig. 4a, it can be seen that both silicon related peaks A and oxide related peak B declined with increasing exposure, however, peak $\mathrm{A}$ decreased at a greater rate, dropping below the intensity of peak B after exposure of $259 \mathrm{nA}$ min. The intensity ratio of peak B (oxide related) over peak A (silicon related) evolved from 0.5 to 1.4 when the exposure increased from 0 to $703 \mathrm{nA}$ min. It was found that the intensity of peak A follows an exponential decay $I_{\mathrm{A}}=18896.7 \times \exp (-X /$ $261.6)+1130$, and that of peak $B$ follows linear regression: $I_{\mathrm{B}}=10486.69464-10.6528 \times X \quad$ (with $\quad R=-0.99101$, $P<0.0001$ perfectly fitted), where $X=$ flux $\times$ time (nA min). These fittings are plotted in Fig. 4b. The exponential decay of peak A intensity is a normal fingerprint of charging phenomena in photoemission spectroscopy [23, 25]. See Fig. 4b, the dotted lines are fittings described above. Very interestingly, during the course of exposure there were no significant changes in the peak positions, see Fig. 4c. From the linear fittings, the positions of both peaks are slightly shifted to higher binding energies: peak A shifted from 99.5 to $99.7 \mathrm{eV}$ while peak B shifted from 102.3 to $102.7 \mathrm{eV}$. And the separation between two peaks is $2.9 \pm 0.1 \mathrm{eV}$. First of all, unlike the alkyl Si-QDs where peak A saturated at $104 \mathrm{eV}$ [25], here peak A was situated at $99.7 \pm 0.2 \mathrm{eV}$ which is in agreement with the real Si2p binding energy of from bulk silicon [46], and results from hydrosilylated $\mathrm{Si}$ (111) surfaces [47]. This can be taken as a measure of a good electrical connection between the sample and substrate with the earth. This can be explained since a thinner and more uniform film was mounted with better contact to the substrate (gold). Another reason could be that the length of acrylic acid coating $\left(\mathrm{CH}_{2}-3\right)$ is much shorter than alkyl coating $\left(\mathrm{CH}_{2}-11\right)$, thus the contact between silicon and substrate is improved. Second, the shifting of peak positions to a higher binding energy is consistent with the decay of the intensities during the irradiation course owing to trapped charges in the sample. Third, the relative quantity of species II and IV in Si2p increased during this photon induced oxidation, thus both peak positions of A and B would be moved towards higher binding energies.

Transmission IR experiments on thermal hydrosilyation of porous silicon shows that the maximum coverage coverage of alkyl chain on porous silicon is $0.3-0.5 \mathrm{ML}$ (monolayer) [48]. This suggests that a substantial fraction of the surface atoms of the Si-QDs are bound to hydrogen. Therefore, the soft X-ray irradiation could induce the formation of oxide species from these sites.

It is reasonable to assign the high binding energy peak B to the photon-induced formation of a number of (sub)oxide species $\left(\mathrm{SiO}_{x}\right.$, where $\left.x \leq 2\right)$. The evolution of the intensity and position of this peak therefore indicates that, as irradiation progress, the coverage of surface oxide and the average oxidation state of the silicon atoms at the surface of the quantum dots increase, which agree well with the sequential appearance of successively higher Si oxidation states during thermally driven oxidation [49].
As the PL profile for Si-QDs is influenced by the degree of oxidation within the nanocrystal structure, the inducement of oxidation by soft X-rays will play a role in the range of potential applications where such materials could be used especially within biomedical labelling. For example, when Si-QDs act as tracers in targeted drug delivery [50], the PL peak position could be changed if X-rays are applied during the process. Consequently the results here provide a very useful reference for future applications.

4 Conclusions In summary, it has been observed for the first time, that irradiation by soft X-ray causes a progression of oxidation within acrylic acid grafted Si-QDs surfaces. Acrylic acid grafted luminescent Si-QDs may be dispersed in water rendering them suitable for biological applications. There are two bands in PL spectrum, originating from $\mathrm{Si}-\mathrm{Si}$ and silicon oxide. The PL band from silicon oxide also indicates the possibility of oxidation under the soft X-ray irradiation. The co-existence of Si2p and C1s core levels confirmed that acrylic acid grafted water soluble silicon nanoparticles have been successfully synthesized. This observation will contribute to the future applications.

Acknowledgements QW is grateful to the International Scholarship Funding panel of the University of East Anglia for awarding an international scholarship. YC is grateful to Royal Society for awarding a Research Grant 2007/R2 and EPSRC for financial support under the project EP/G01664X/1. The research leading to these results has received funding from the European Community's Seventh Framework Programme (FP7/2007-2013) under grant agreement $n^{\circ} 226716$.

\section{References}

[1] L. T. Canham, Appl. Phys. Lett. 57, 1046 (1990).

[2] D. Moore, S. Krishnamurthy, Y. Chao, Q. Wang, D. Brabazon, and P. J. McNally, Phys. Status Solidi A 208, 604 (2011).

[3] X. Michalet, F. F. Pinaud, L. A. Bentolila, J. M. Tsay, S. Doose, J. J. Li, G. Sundaresan, A. M. Wu, S. S. Gambhir, and S. Weiss, Science 307, 538 (2005).

[4] A. I. Boukai, Y. Bunimovich, J. Tahir-Kheli, J. K. Yu, W. A. Goddard, and J. R. Heath, Nature 451, 168 (2008).

[5] A. I. Hochbaum, R. K. Chen, R. D. Delgado, W. J. Liang, E. C. Garnett, M. Najarian, A. Majumdar, and P. D. Yang, Nature 451, 163 (2008).

[6] T. P. Burg, M. Godin, S. M. Knudsen, W. Shen, G. Carlson, J. S. Foster, K. Babcock, and S. R. Manalis, Nature 446, 1066 (2007).

[7] J. F. Li, Y. F. Huang, Y. Ding, Z. L. Yang, S. B. Li, X. S. Zhou, F. R. Fan, W. Zhang, Z. Y. Zhou, D. Y. Wu, B. Ren, Z. L. Wang, and Z. Q. Tian, Nature 464, 392 (2010).

[8] L. Bagolini, A. Mattoni, G. Fugallo, L. Colombo, E. Poliani, S. Sanguinetti, and E. Grilli, Phys. Rev. Lett. 104, 176803 (2010).

[9] S. Polisski, B. Goller, K. Wilson, D. Kovalev, V. Zaikowskii, and A. Lapkin, J. Catal. 271, 59 (2010).

[10] L. Šiller, S. Krishnamurthy, L. Kjeldgaard, B. R. Horrocks, Y. Chao, A. Houlton, A. K. Chakraborty, and M. R. C. Hunt, J. Phys.: Condens. Matter 21, 095005 (2009).

[11] R. J. Rostron, Y. Chao, G. Roberts, and B. R. Horrocks, J. Phys.: Condens. Matter 21, 8 (2009). 
[12] K. Fujioka, M. Hiruoka, K. Sato, N. Manabe, R. Miyasaka, S. Hanada, A. Hoshino, R. D. Tilley, Y. Manome, K. Hirakuri, and K. Yamamoto, Nanotechnology 19, 415102 (2008).

[13] N. H. Alsharif, C. E. M. Berger, S. S. Varanasi, Y. Chao, B. R. Horrocks, and H. K. Datta, Small 5, 221 (2009).

[14] R. S. Carter, S. J. Harley, P. P. Power, and M. P. Augustine, Chem. Mater. 17, 2932 (2005).

[15] Z. F. Li and E. Ruckenstein, Nano Lett. 4, 1463 (2004).

[16] S. Sato and M. T. Swihart, Chem. Mater. 18, 4083 (2006).

[17] S. W. Lin and D. H. Chen, Small 5, 72 (2009).

[18] R. D. Tilley and K. Yamamoto, Adv. Mater. 18, 2053 (2006).

[19] J. H. Warner, A. Hoshino, K. Yamamoto, and R. D. Tilley, Angew. Chem. Int. Ed. 44, 4550 (2005).

[20] J. G. Franchina, W. M. Lackowski, D. L. Dermody, R. M. Crooks, and D. E. Bergbreiter, Anal. Chem. 71, 3133 (1999).

[21] Z. F. Li, E. T. Kang, K. G. Neoh, and K. L. Tan, Biomaterials 19, 45 (1998).

[22] L. H. Lie, M. Duerdin, E. M. Tuite, A. Houlton, and B. R. Horrocks, J. Electroanal. Chem. 538, 183 (2002).

[23] T. Tamura and S. Adachi, J. Appl. Phys. 105, 113518 (2009).

[24] I. D. Sharp, Q. Xu, C. W. Yuan, J. W. Beeman, J. W. Ager, D. C. Chrzan, and E. E. Haller, Mater. Res. Soc. Symp. Proc. 958, 87 (2007).

[25] Y. Chao, S. Krishnamurthy, M. Montalti, L. Šiller, L. H. Lie, A. Houlton, B. R. Horrocks, L. Kjeldgaard, V. R. Dhanak, and M. R. C. Hunt, J. Appl. Phys. 98, 044316 (2005).

[26] Y. Chao, A. Houlton, B. R. Horrocks, M. R. C. Hunt, N. R. J. Poolton, J. Yang, and L. Šiller, Appl. Phys. Lett. 88, 263119 (2006).

[27] M. Ishii, B. Hamilton, N. R. J. Poolton, N. Rigopoulos, S. De Gendt, and K. Sakurai, Appl. Phys. Lett. 90, 063101 (2007).

[28] A. V. Osipov, P. Patzner, and P. Hess, Appl. Phys. A 82, 275 (2006).

[29] Q. Wang, H. Ni, A. Pietzsch, F. Hennies, Y. Bao, and Y. Chao, J. Nanoparticle Res. 13, 405 (2011).

[30] A. Uhlir, Bell Syst. Tech. J. 35, 333 (1956).

[31] A. Hadjadj, N. Pham, P. R. I. Cabarrocas, O. Jbara, and G. Djellouli, J. Appl. Phys. 107, 083509 (2010).

[32] R. Denecke, P. Vaterlein, M. Bassler, N. Wassdahl, S. Butorin, A. Nilsson, J. E. Rubensson, J. Nordgren, N. Martensson, and R. Nyholm, J. Electron Spectrosc. Relat. Phenom. 103, 971 (1999).
[33] Y. Chao, L. Šiller, S. Krishnamurthy, P. R. Coxon, U. Bangert, M. Gass, L. Kjeldgaard, S. N. Patole,O L. H. Lie, N. O'Farrell, T. A. Alsop, A. Houlton, and B. R. Horrocks, Nature Nanotechnol. 2, 486 (2007).

[34] D. A. Dixon and J. L. Gole, J. Phys. Chem. B 109, 14830 (2005).

[35] S. V. Gaponenko, E. P. Petrov, U. Woggon, O. Wind, C. Klingshirn, Y. H. Xie, I. N. Germanenko, and A. P. Stupak, J. Lumin. 70, 364 (1996).

[36] M. V. Wolkin, J. Jorne, P. M. Fauchet, G. Allan, and C. Delerue, Phys. Rev. Lett. 82, 197 (1999).

[37] C. Itoh, K. Tanimura, N. Itoh, and M. Itoh, Phys. Rev. B 39, 11183 (1989).

[38] T. K. Sham, S. J. Naftel, P. S. G. Kim, R. Sammynaiken, Y. H. Tang, I. Coulthard, A. Moewes, J. W. Freeland, Y. F. Hu, and S. T. Lee, Phys. Rev. B 70, 045313 (2004).

[39] X. G. Li, Y. Q. He, S. S. Talukdar, and M. T. Swihart, Langmuir 19, 8490 (2003).

[40] M. H. Nayfeh, N. Barry, J. Therrien, O. Akcakir, E. Gratton, and G. Belomoin, Appl. Phys. Lett. 78, 1131 (2001).

[41] S. Komuro, T. Kato, T. Morikawa, P. Okeeffe, and Y. Aoyagi, J. Appl. Phys. 80, 1749 (1996).

[42] C. D. Wanger, W. M. Riggs, L. E. Davis, J. F. Moulder, and G. E. Muilenberg, Handbook of X-ray Photoelectron Spectroscopy (Perkin Elmer Corporation, Eden Prairie, Minnesota, 1979).

[43] Z. L. Liu, J. F. Liu, P. Ren, Y. Y. Wu, and P. S. Xu, Appl. Surf. Sci. 254, 3207 (2008).

[44] J. H. Park, W. C. Mitchel, L. Grazulis, K. Eyink, H. E. Smith, and J. E. Hoelscher, Carbon 49, 631 (2011).

[45] S. R. Puniredd, O. Assad, and H. Haick, J. Am. Chem. Soc. 130, 9184 (2008).

[46] A. C. Thompson and V. Douglas, X-ray Data Booklet (Lawrence Berkeley National Lab, Berkeley, 2001).

[47] A. Lehner, G. Steinhoff, M. S. Brandt, M. Eickhoff, and M. Stutzmann, J. Appl. Phys. 94, 2289 (2003).

[48] A. B. Sieval, B. van den Hout, H. Zuilhof, and E. J. R. Sudholter, Langmuir 17, 2172 (2001).

[49] A. Yoshigoe, K. Moritani, and Y. Teraoka, Appl. Surf. Sci. 216, 388 (2003).

[50] V. Biju, T. Itoh, and M. Ishikawa, Chem. Soc. Rev. 16, 1186 (2010). 